\title{
Comparison of Path Integral Monte Carlo Simulations of Helium, Carbon, Nitrogen, Oxygen, Water, Neon, and Silicon Plasmas
}

\author{
K. P. Driver ${ }^{\mathrm{a}}$, Francois Soubiran ${ }^{\mathrm{a}}$, Shuai Zhang ${ }^{\mathrm{a}}$, B. Militzer ${ }^{\mathrm{a}, \mathrm{b}}$ \\ ${ }^{a}$ Department of Earth and Planetary Science, University of California, Berkeley, California 94720, USA \\ ${ }^{b}$ Department of Astronomy, University of California, Berkeley, California 94720, USA
}

\begin{abstract}
We have performed all-electron path integral Monte Carlo (PIMC) and density functional theory molecular dynamics (DFT-MD) calculations to explore properties of first- and second-row materials in the liquid, warm dense matter, and plasma regimes. Our simulations have covered a wide density-temperature range of roughly $1-15 \mathrm{~g} \mathrm{~cm}^{-3}$ and $10^{4}-10^{9} \mathrm{~K}$ ). We first analyze the ionization behavior of carbon and water plasma. Then we provide a comparative analysis of the pair-correlation functions and Hugoniot curves of $\mathrm{He}, \mathrm{C}, \mathrm{N}, \mathrm{O}, \mathrm{Ne}$, and Si plasmas. Pair-correlation functions give insight into the evolution of plasma structure and ionization processes that are driven by changes in temperature and density. Finally, we show that the maximum shock compression of a material is controlled by the ionization of L-shell and K-shell electrons and depends strongly on this as a function of the atomic number of the material.
\end{abstract}

Keywords: Path Integral Monte Carlo, Warm Dense Matter, Pair-correlation, Shock Hugoniot

\section{Introduction}

A rigorous and consistent theoretical description of materials residing in the high energy density physics (HEDP) and warm dense matter (WDM) regimes has been identified [1, 2, 3] as a central goal to the development of key plasma technologies, such as inertial and magnetically confined fusion, shock physics, high energy astrophysics, and stockpile stewardship. Some of the most important pieces of information theoretical calculations can provide include the equation of state (EOS), pair-correlation functions, and transport and optical properties. These quantities provide fundamental information from which many other physical properties can be derived and, subsequently, used to build theoretical models and design HEDP experiments. For example, the design of a fusion experiment [4] relies heavily on understanding how plasma constituents evolve with temperature and density in order to optimize ignition parameters. In addition, knowledge of the plasma dependence on temperature and density provides key constraints on input parameters in hydrodynamic simulations, which are critical in the designs of experiments.

Development of a comprehensive first-principles framework that can accurately and reliably predict plasma properties across the entire WDM regime remains a significant challenge. While semi-analytic plasma models [5] are sufficient for describing weak-to-moderate coupling regimes, first-principles-based methods, such as path integral Monte Carlo (PIMC) [6], orbital-free density functional theory

Email address: kdriver@berkeley.edu (K. P. Driver)

URL: http://militzer.berkeley.edu/ driver/ (K. P. Driver)

Preprint submitted to Elsevier
(OF-DFT) [7, 8, 9], and average-atom methods [10, 11, 12], are needed to accurately address the strong coupling regimes along the pathway to fusion. In the WDM regime, materials are only partially ionized, and electrons are still strongly correlated such that quantum effects (quantum degeneracy, ionization, and dissociation) are all relevant for determining plasma properties [13].

PIMC is uniquely suited to provide benchmark-quality predictions in the WDM regime, because it is the only method which is able to treat all of the quantum effects appropriately by solving the full finite-temperature quantum many-body problem. The challenge in PIMC is to further develop the technique for simulations of heavier elements, which we have been working towards for the past several years. We have successively applied PIMC to study progressively higher $\mathrm{Z}$ materials in the liquid-, warm-, and plasma regimes, namely hydrogen [14], helium 15], carbon [16], nitrogen [17], water [16], oxygen [18], neon [19], sodium [20] and silicon [21]. As part of this work, we have significantly developed the capability of PIMC to utilize nodal surfaces beyond that of free-particles, which will pave the way for PIMC simulations of heavy elements in the WDM regime. For these materials, we have also performed Kohn-Sham DFT-MD simulations at lower temperatures where most electrons reside in bound states in order to provide one coherent EOS table that covers a wide range of temperature and density conditions.

In this paper, we provide additional analyses of previously published PIMC simulations on low-Z elements. In Section 2, we briefly summarize the details of the simulation methods. In Section 3, we present pair-correlation data from our simulations of carbon and water plasmas. 
In Section 4, we compare our set of pair-correlation function data for low-Z elements and discuss common trends. In section 5, we compare our set of shock Hugoniot curves for low-Z elements and discuss common trends. Finally, in Section 6, we provide some conclusions.

\section{Simulation Methods}

Rigorous discussions of the PIMC [22, 23, 24] and DFT molecular dynamics (DFT-MD) [25] methods have been provided in previous works, and the details of our simulations have been presented in our previous publications [14, 15, 16, 21]. Here, we simply summarize the methods and choices we make to set the parameters of the calculations.

PIMC is a state-of-the-art first-principles method for computing the properties of interacting quantum systems at finite temperature. The method involves stochastically solving for the thermal density matrix, expressed in imaginary time path integrals, which is directly related to the partition function and thermodynamic observables. This framework provides an explicit many-body method that treats all the important physical effects in the WDM regime, such as bonding, ionization, exchange-correlation, and quantum degeneracy 26. The Coulomb interaction is incorporated via pair density matrices derived from the eigenstates of the two-body Coulomb problem [27, 28, 29]. The efficiency of PIMC increases with temperature as paths get shorter and particles behave more classically.

In order to simulate fermions, we restrict the paths in the fixed-node approximation to avoid the fermion sign problem [30]. We have found approximate, free-particle nodal surfaces are sufficient to simulate first-row plasmas at temperatures as low as $1 \times 10^{6} \mathrm{~K}$. Free particle nodes are sufficient at temperatures where nearly all states except 1s states are ionized [16]. In order to go beyond the freeparticle nodal approximation, we have recently developed a localized nodal surface to treat bound states in secondrow elements [21].

PIMC uses a small number of controlled approximations, whose errors can be minimized by converging parameters, such as the time step and system size. In simulations using free particle nodes, we typically use a time-step $(\tau$ $\left.=\frac{1}{N_{\text {slices }} k_{\mathrm{B}} T}\right)$ of $1 / 256 \mathrm{Ha}^{-1}(1 \mathrm{Ha}=27.211396 \mathrm{eV})$ for temperatures below $4 \times 10^{6} \mathrm{~K}$, where $\mathrm{N}_{\text {slices }}$ is the number of discretized time slices associated with convolutions of the density matrix. This choice converges the total energy per atom within 1\%. For higher temperatures, we typically decrease the time step as $1 / \mathrm{T}$, using between 4 and 80 time slices. For simulations using localized nodal surfaces, we use a much smaller time-step of $1 / 8192 \mathrm{Ha}^{-1}$, using between 20 and 2560 time slices. Regarding finite size errors, we showed simulations of 8 - and 24-atom cubic cells provide total energies that agree within $0.4 \%$ for the relevant temperatures [19]. Our results for the internal energy and pressure typically have statistical errors of $0.3 \%$ or less. All pair-correlation functions shown here are based on simulations cells comprised of 8 formula units (f.u.).
While PIMC simulations are accurate and increasingly efficient at high temperatures $\left(\mathrm{T}>1 \times 10^{6} \mathrm{~K}\right)$, DFT-MD provides an efficient, state-of-the-art, first-principles method at lower temperatures $\left(\mathrm{T}<1 \times 10^{6} \mathrm{~K}\right)$. The DFT formalism maps the many-body problem onto a single-particle problem with an approximate exchange-correlation potential. In the WDM regime, where temperatures are at or above the Fermi temperature, the exchange-correlation functional is not explicitly designed to accurately describe the electronic excitations [31]. However, in our PIMC and DFT-MD studies of first- and second-row elements, we found zero-temperature DFT functionals predicted energies within 2-5 Ha/atom of our PIMC results at temperatures near $1 \times 10^{6} \mathrm{~K}$. This suggests that the error that is introduced by using a ground-state functional is rather small.

DFT incorporates effects of finite electronic temperature by using a Fermi-Dirac function to smear out the thermal occupation of single-particle electronic states [32]. As temperature grows large, an increasing number of bands are required to account for the occupation of excited states in the continuum, which typically causes the efficiency of the algorithm to become intractable at temperatures beyond $1 \times 10^{6} \mathrm{~K}$, where on the order of a thousand orbitals per atom are partially occupied. In addition, pseudopotentials typically replace the core electrons in each atom to improve efficiency. Here, we are careful to avoid using DFT-MD at temperatures where the $\mathrm{K}$ shell electrons undergo excitations and study those conditions with PIMC instead.

We employ standard Kohn-Sham DFT-MD simulations for our calculations in the WDM regime. We use the Vienna Ab initio Simulation Package (VASP) 33] with the projector augmented-wave (PAW) method [34], and a NVT ensemble (i.e., constant number of particles, N, volume, V, and temperature, T) regulated with a Nosé thermostat. Exchange-correlation effects are described using the Perdew-Burke-Ernzerhof [35] generalized gradient approximation. Electronic wave functions are expanded in a plane-wave basis with an energy cut-off as high as $4000 \mathrm{eV}$ in order to converge total energy. Size convergence tests up to a 24-f.u. simple cubic simulation cell at temperatures of $10,000 \mathrm{~K}$ and above indicate that total energies are converged to better than $0.1 \%$. We find, at temperatures above 250,000 K, 8-f.u. supercell results are sufficient since the kinetic energy far outweighs the interaction energy at such high temperatures 19]. The number of bands in each calculation is selected such that thermal occupation is converged to better than $10^{-4}$, which requires up to 10,000 bands in a 8 -f.u. cell at $1 \times 10^{6} \mathrm{~K}$. All simulations are performed at the $\Gamma$-point of the Brillouin zone, which is sufficient for high temperature fluids, converging total energy to better than $0.01 \%$ relative to a comparison with a converged grid of k-points. 


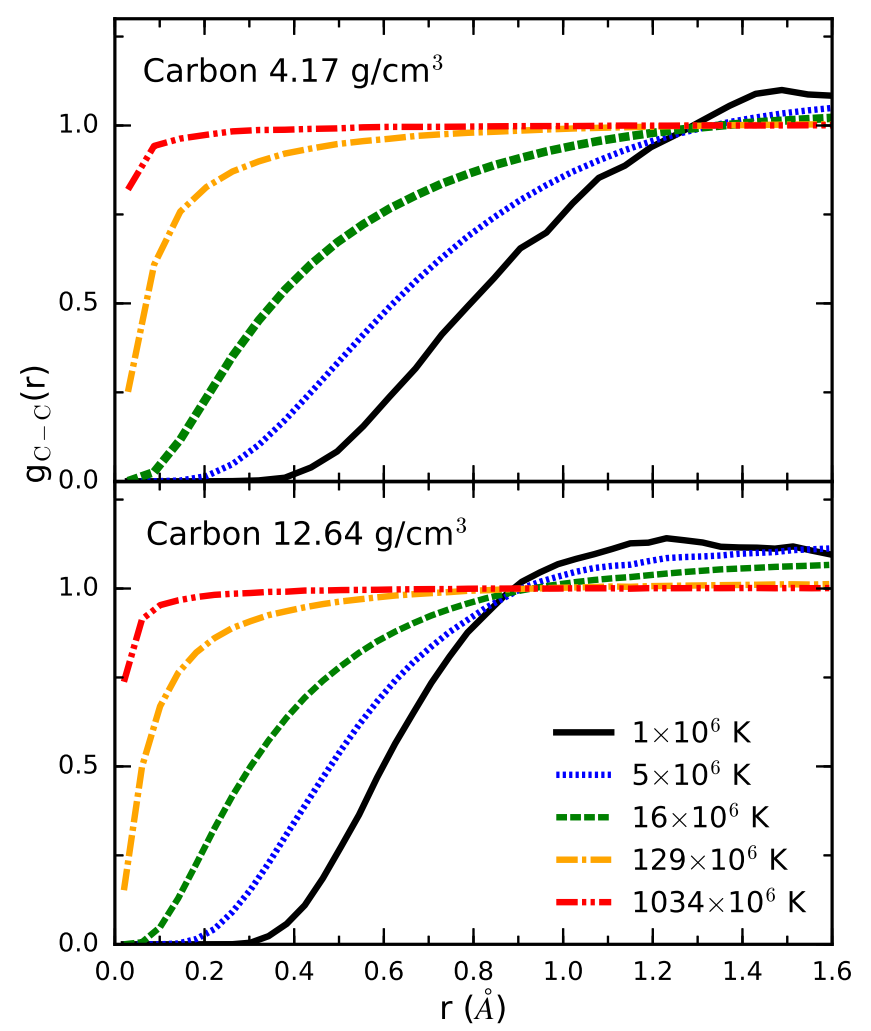

Figure 1: C-C ion pair-correlation functions from PIMC simulations of carbon plasma.

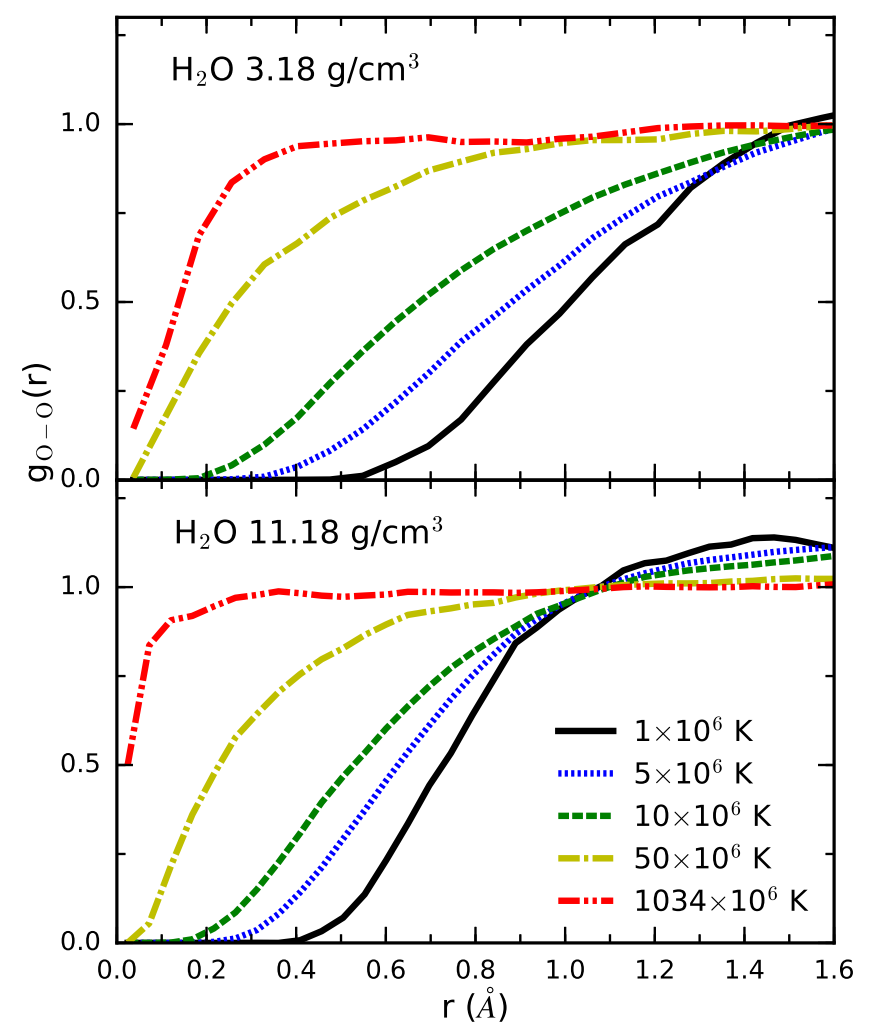

Figure 2: $\mathrm{O}-\mathrm{O}$ ion pair-correlation functions from PIMC simulations of $\mathrm{H}_{2} \mathrm{O}$ plasma.

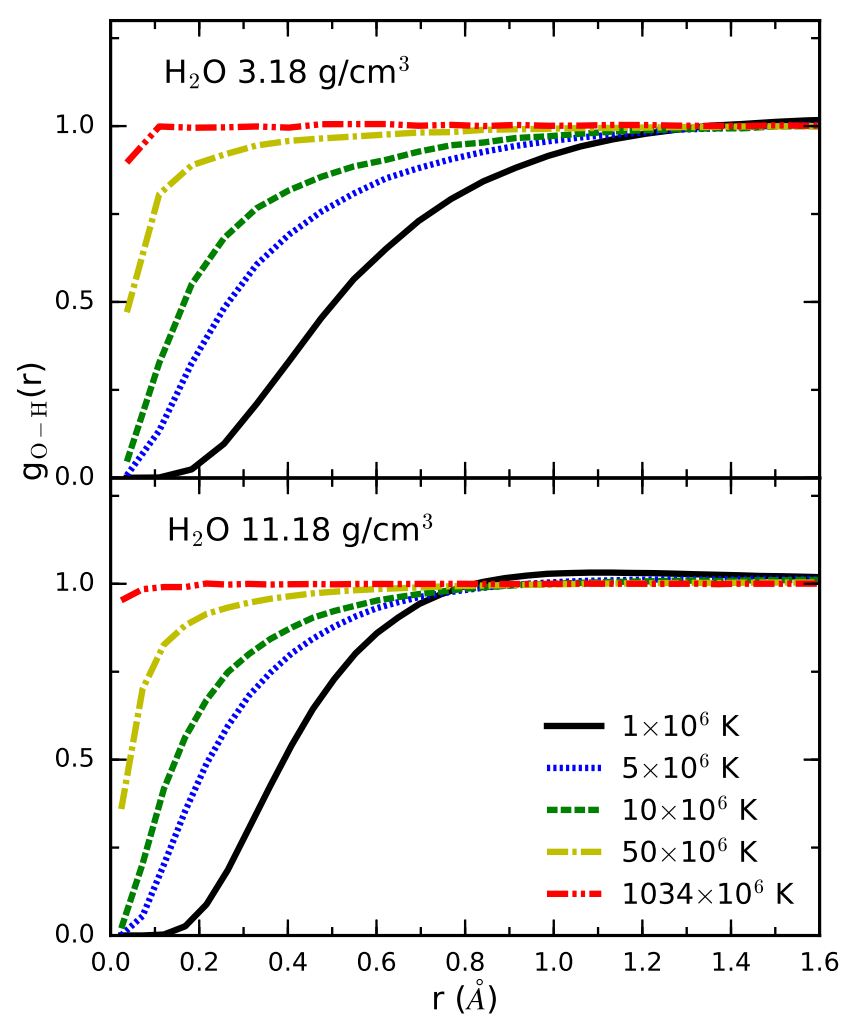

Figure 3: O-H ion pair-correlation functions from PIMC simulations of $\mathrm{H}_{2} \mathrm{O}$ plasma.

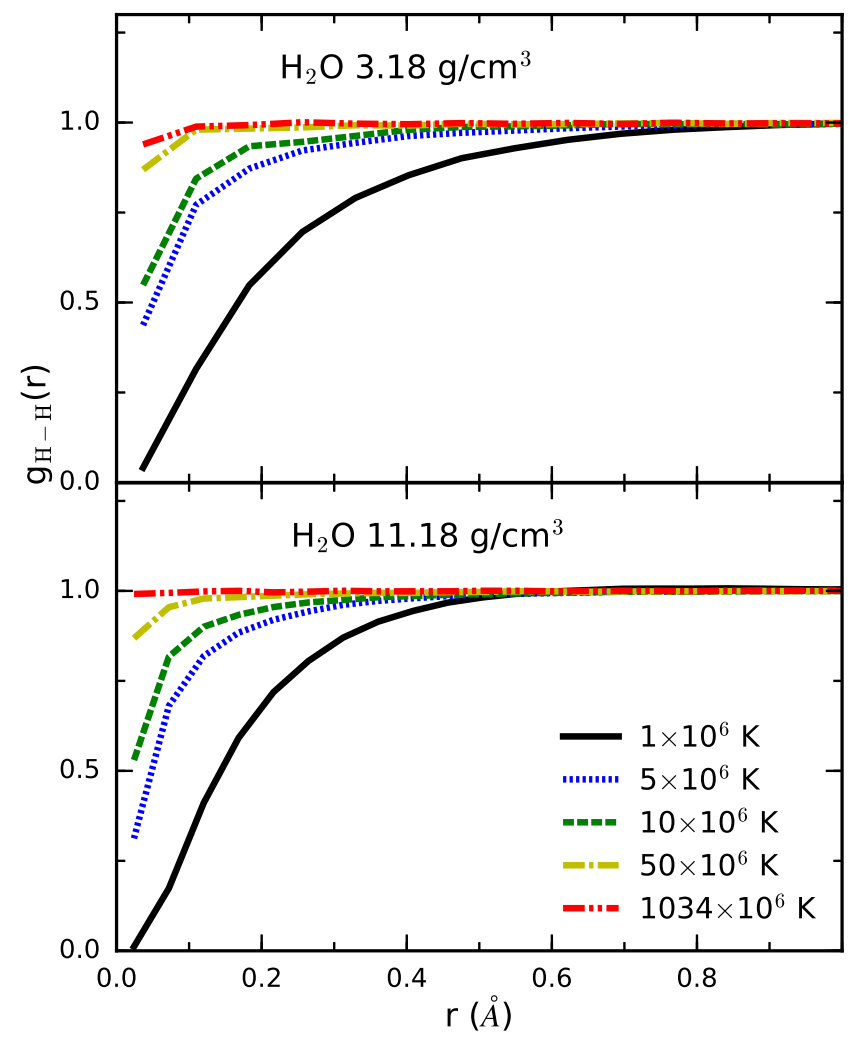

Figure 4: $\mathrm{H}-\mathrm{H}$ pair-correlation functions from PIMC simulations of $\mathrm{H}_{2} \mathrm{O}$ plasma. 


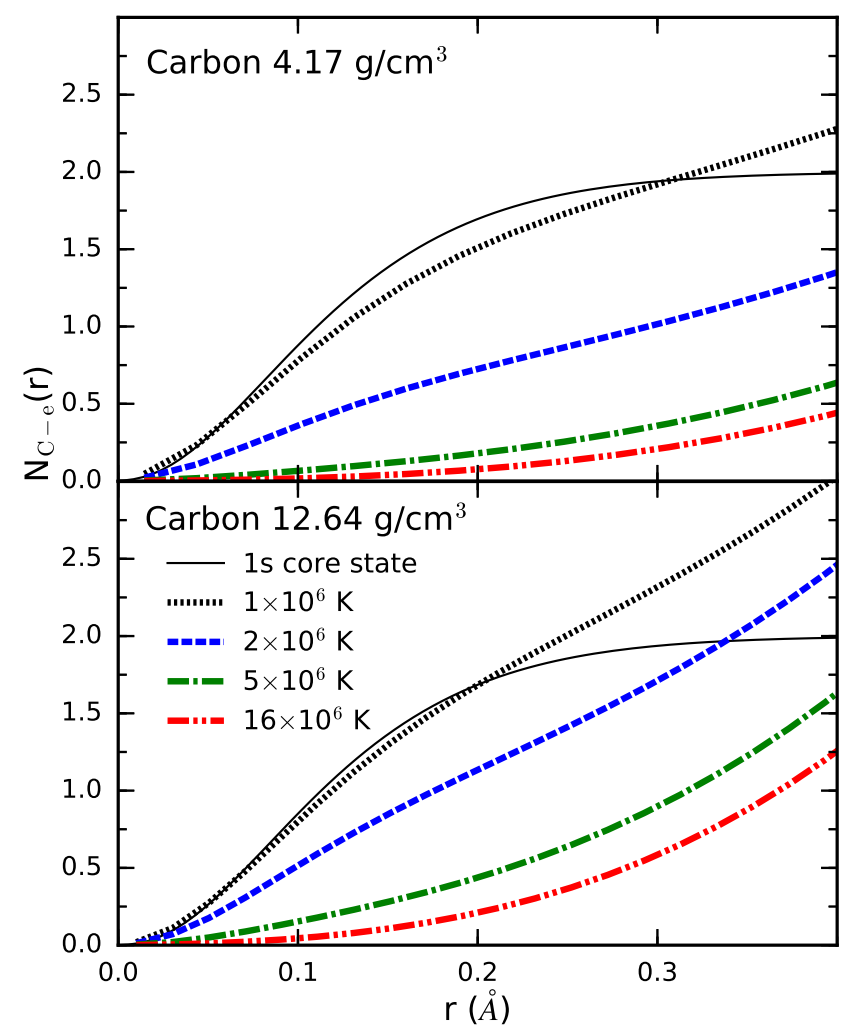

Figure 5: Number of electrons contained in a sphere of radius, $\mathrm{r}$, around a carbon nucleus in PIMC simulations of carbon plasma.

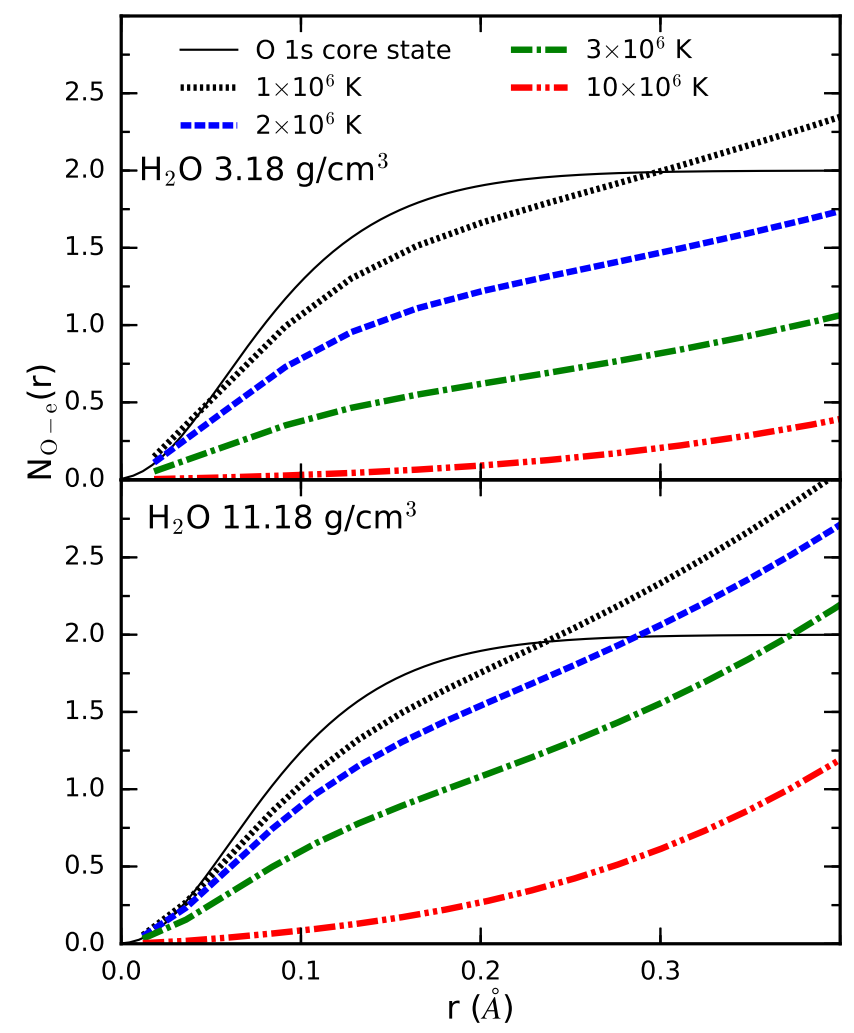

Figure 6: Number of electrons contained in a sphere of radius, $r$, around an oxygen nucleus in PIMC simulations of $\mathrm{H}_{2} \mathrm{O}$ plasma.

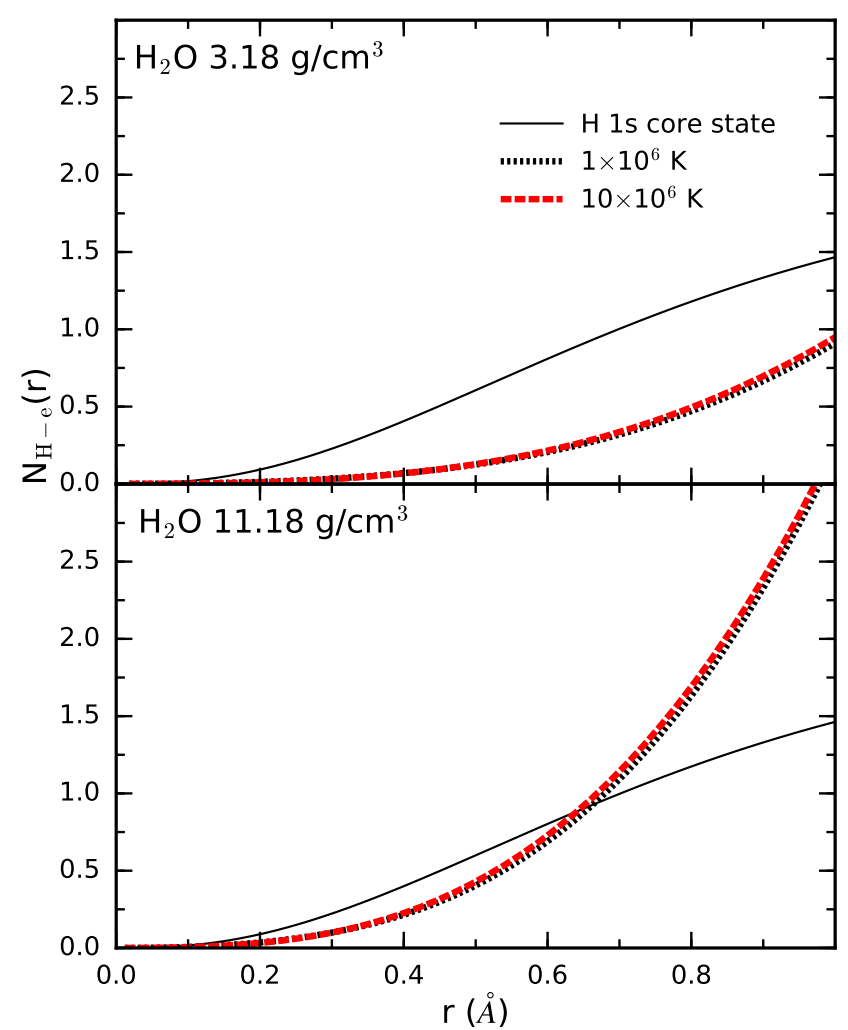

Figure 7: Number of electrons contained in a sphere of radius, r, around a hydrogen nucleus in PIMC simulations of $\mathrm{H}_{2} \mathrm{O}$ plasma. 


\section{Pair-correlation functions for carbon and $\mathrm{H}_{2} \mathrm{O}$}

Our initial publication on PIMC simulations of carbon and water plasmas [16] provided the equation of state across the WDM regime and showed that PIMC and DFTMD produce the same ionic plasma structure. Here, we provide a discussion of the temperature and density dependence of pair-correlation functions and ionization processes in warm dense carbon and water. The pair correlation is defined as

$$
g(r)=\frac{V}{N^{2}}\left\langle\sum_{i} \sum_{j \neq i} \delta\left(r-r_{i j}\right)\right\rangle,
$$

where $\mathrm{N}$ is the total number of particles, $\mathrm{V}$ is the cell volume, and $\mathrm{r}$ is the distance from the ith reference particle. Results discussed below are based on 8-atom (carbon) or 8-molecule (water) simulation cells.

Figures 1 , 2] 3, and 4 show ion-ion pair-correlation functions, $g(r)$, for $\mathrm{C}-\mathrm{C}$ pairs in carbon plasma and $\mathrm{O}-\mathrm{O}$, $\mathrm{O}-\mathrm{H}$, and $\mathrm{H}-\mathrm{H}$ pairs in water plasmas, respectively. The $\mathrm{g}(\mathrm{r})$ functions were computed with PIMC over a temperature range of $1 \times 10^{6}-1.034 \times 10^{9} \mathrm{~K}$ at densities of 4.17 and $12.64 \mathrm{~g} \mathrm{~cm}^{-3}$ for carbon and densities of 3.18 and 11.18 $\mathrm{g} \mathrm{cm}^{-3}$ for water. In each case, the atoms are kept farthest apart at low temperatures due to a combination of Pauli exclusion among bound electrons and Coulomb repulsion. As temperature increases, kinetic energy of the nuclei increases, making it more likely to find atoms at close range. At the same time, the atoms become increasingly ionized, which gradually reduces the Pauli repulsion, but increases the ionic Coulomb repulsion. As density increases, the likelihood of finding two nuclei at close range rises slightly. At high temperatures, the system approaches the DebyeHückel limit, behaving like a weakly correlated system of screened Coulomb charges.

Figures 5and 6 show the integral of the nucleus-electron pair correlation function, $N(r)$, for C-e in carbon plasma, and O-e water plasma, respectively. $\mathrm{N}(\mathrm{r})$ is given by the formula

$$
N(r)=\left\langle\frac{1}{N_{I}} \sum_{e, I} \theta\left(r-\left|\vec{r}_{e}-\vec{r}_{I}\right|\right)\right\rangle,
$$

where the sum includes all electron-ion pairs and $\theta$ represents the Heaviside function. N(r) represents the average number of electrons within a sphere of radius $r$ around a given nucleus. From the comparison with 1s ground state of an isolated atom, we find that the 1s sates in our PIMC simulations are only slightly ionized at the lowest temperature of $1 \times 10^{6} \mathrm{~K}$. As temperature increases, the states are further ionized and most electrons become unbound, causing $N(r)$ to decrease. At higher density, an even higher temperature is required to fully ionize the atoms, indicating that the 1s ionization fraction decreases with density and pressure ionization is absent, as discussed in detail in our previous paper [18]. Analogously, we plot $N(r)$ for H-e pairs in Fig. 7. which simply shows the hydrogen atom is fully ionized at these temperatures, and, at higher densities, more electrons are found at closer range to the ions.

Figures 8 and 9 show electron-electron pair correlations in carbon and water plasma, respectively, for electrons having opposite spins. The functions are multiplied by the number density $\rho$, so that the integral under the curves is proportional to the number of electrons. The electrons are most highly correlated for low temperatures, which reflects that multiple electrons occupy bound states around a given nucleus. As temperature increases, electrons are thermally excited, decreasing the correlation among each other. The positive correlation at short distances increases with density, consistent with a lower ionization fraction.

Figures 10 and 11] show electron-electron pair correlations in carbon and water plasma, respectively, for electrons with parallel spins. The positive correlation at intermediate distances reflects that different electrons with parallel spins are bound to a given nucleus. For short separations, electrons strongly repel due to Pauli exclusion and the functions decay to zero. As density increases, the peak at intermediate distances decreases, which clearly shows the effect of pressure ionization of the L shell. Pressure ionization is expected for these orbitals because they are much larger than the 1s orbitals and are therefore subject to Pauli exchange with nearby nuclei. As temperature increases, electrons become less bound, which also causes the correlation to become more like an ideal fluid.

\section{Comparison of pair-correlation functions}

Figure 12 compares PIMC ion-ion pair-correlation functions, $g(r)$, for $\mathrm{C}, \mathrm{N}, \mathrm{O}, \mathrm{Ne}$, and $\mathrm{Si}$ plasmas at temperatures of 1 and $2 \times 10^{6} \mathrm{~K}$ and similar densities. Lighter ions are systematically found at closer range than heavier ions due to smaller Coulomb repulsion and kinetic effects. In each system, the atoms are kept farther apart at low temperatures and found closer together at high temperature due to stronger collisions and reduced Pauli exclusion effects. As density increases, we see a slight increase in correlation at short distances in each case. PIMC and DFT-MD predict consistent $\mathrm{g}(\mathrm{r})$ curves in the region of temperature-density space results can overlap. For example, in the figure, we compare the $\mathrm{g}(\mathrm{r})$ curves from PIMC and DFT for silicon at $9.3 \mathrm{~g} \mathrm{~cm}^{-3} 1 \times 10^{6} \mathrm{~K}$. Our previous publications [16, 18, 19, 17] have shown PIMC and DFT-MD predict consistent ionic $\mathrm{g}(\mathrm{r})$ results for the other materials at similar conditions. 


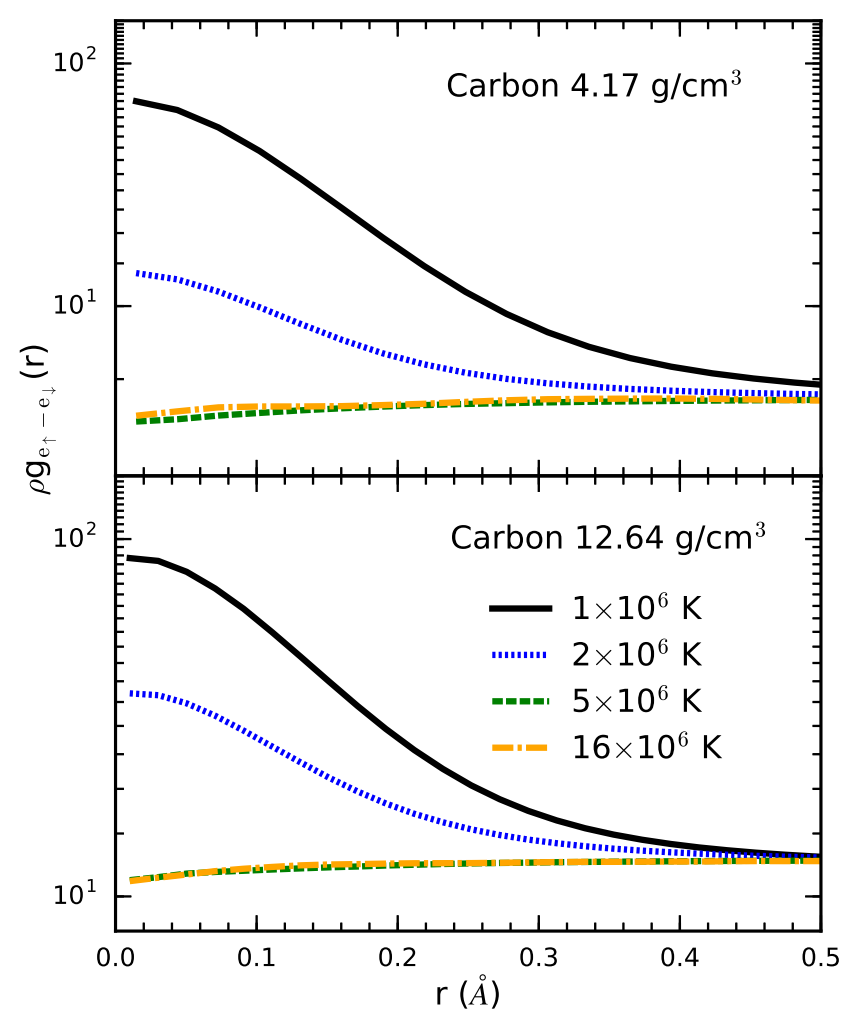

Figure 8: The electron-electron pair-correlation functions for electrons with anti-parallel spins computed in PIMC calculations of carbon plasma.

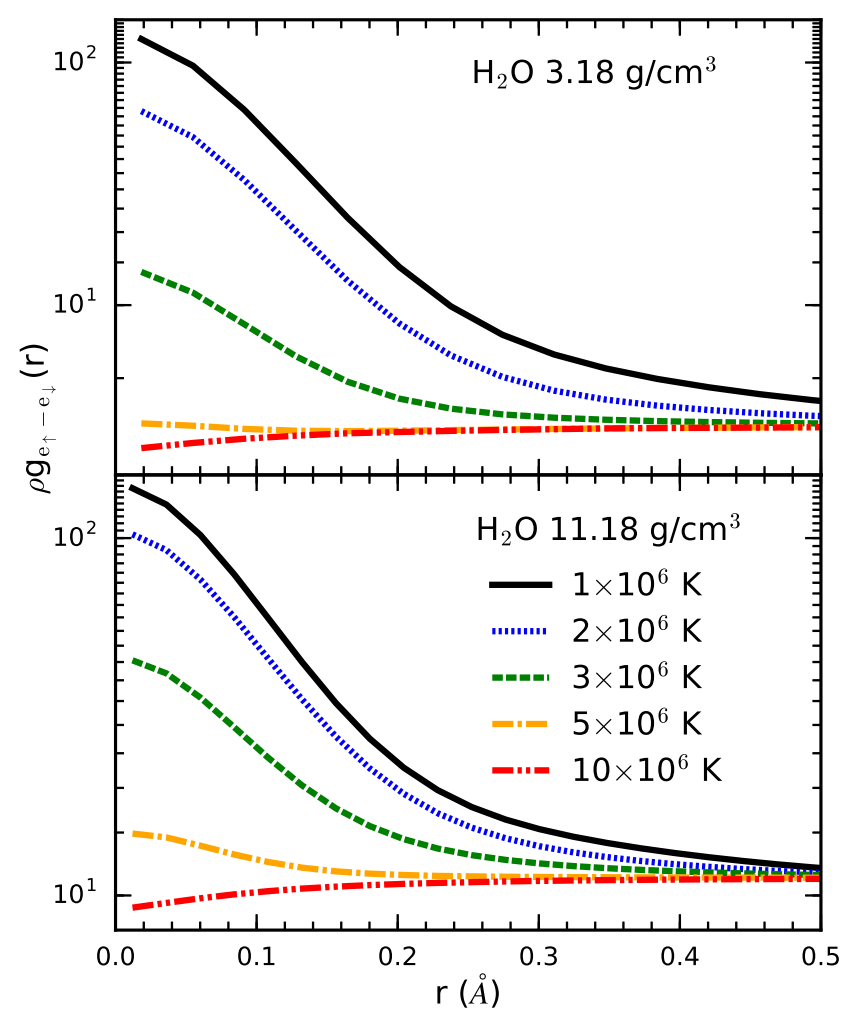

Figure 9: The electron-electron pair-correlation functions for electrons with anti-parallel spins in PIMC calculations of $\mathrm{H}_{2} \mathrm{O}$ plasma.

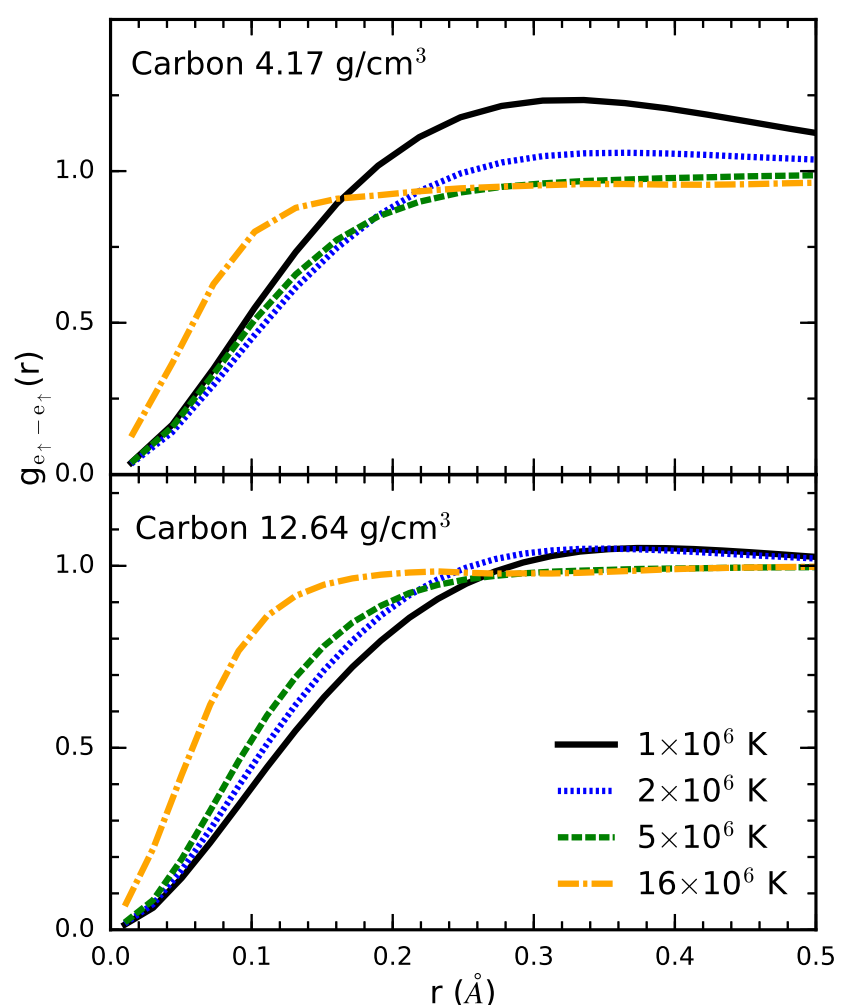

Figure 10: The electron-electron pair-correlation functions for electrons with parallel spins in PIMC calculations of carbon plasma.

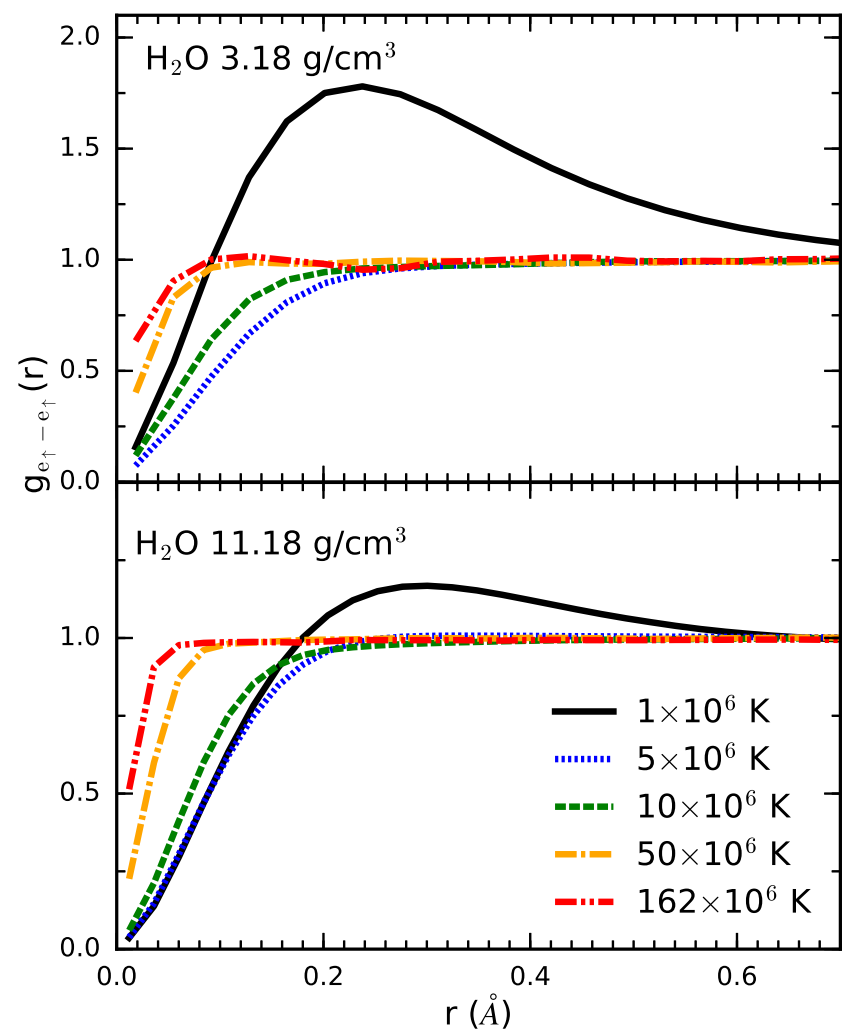

Figure 11: The electron-electron pair-correlation functions for electrons with parallel spins in PIMC calculations of $\mathrm{H}_{2} \mathrm{O}$ plasma. 


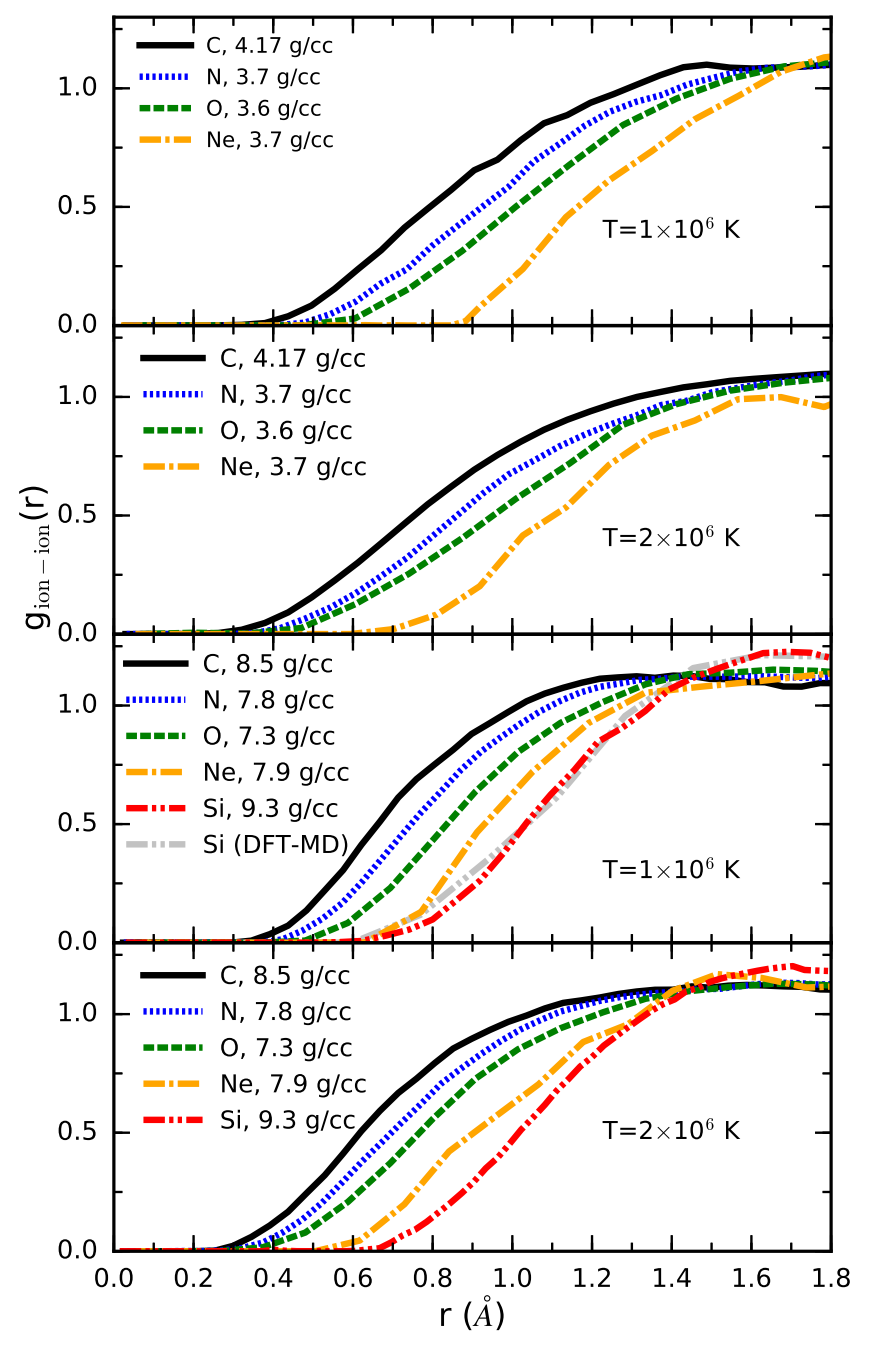

Figure 12: Ion-ion pair-correlation functions from PIMC calculations of various plasmas at two sets of temperatures and densities. The gray curve at $9.3 \mathrm{~g} \mathrm{~cm}^{-3}$ and $1 \times 10^{6} \mathrm{~K}$ is the DFT-MD result for silicon, which agrees well with the PIMC result at the same conditions.

Figure 13 shows the $N(r)$ functions for $\mathrm{C}, \mathrm{N}, \mathrm{O}, \mathrm{Ne}$, and $\mathrm{Si}$ at temperatures of 1 and $4 \times 10^{6} \mathrm{~K}$ at similar densities. As temperature increases in each system, the $\mathrm{N}(\mathrm{r})$ function decreases due to an increased fraction of thermal ionization. The ionization fraction strongly depends on the atomic number, $\mathrm{Z}$, since the electronic binding energy scales at $Z^{2}$, requiring higher temperatures to ionize core electrons in higher $\mathrm{Z}$ atoms. At $1 \times 10^{6} \mathrm{~K}$, all systems have at least partially bound 1 s electrons based on comparison with the corresponding 1 s core state, while at $4 \times 10^{6} \mathrm{~K}$, only $\mathrm{Si}$ and $\mathrm{Ne}$ still have bound 1 s electrons. As density increases, the ionization fraction decreases in each system, but the effect is less significant for core electrons in higher Z systems within the density-temperature range under consideration. There is no sign of pressure ionization in any system at the conditions considered.

For C, N, O, Ne, and Si plasmas, Figure 14 show

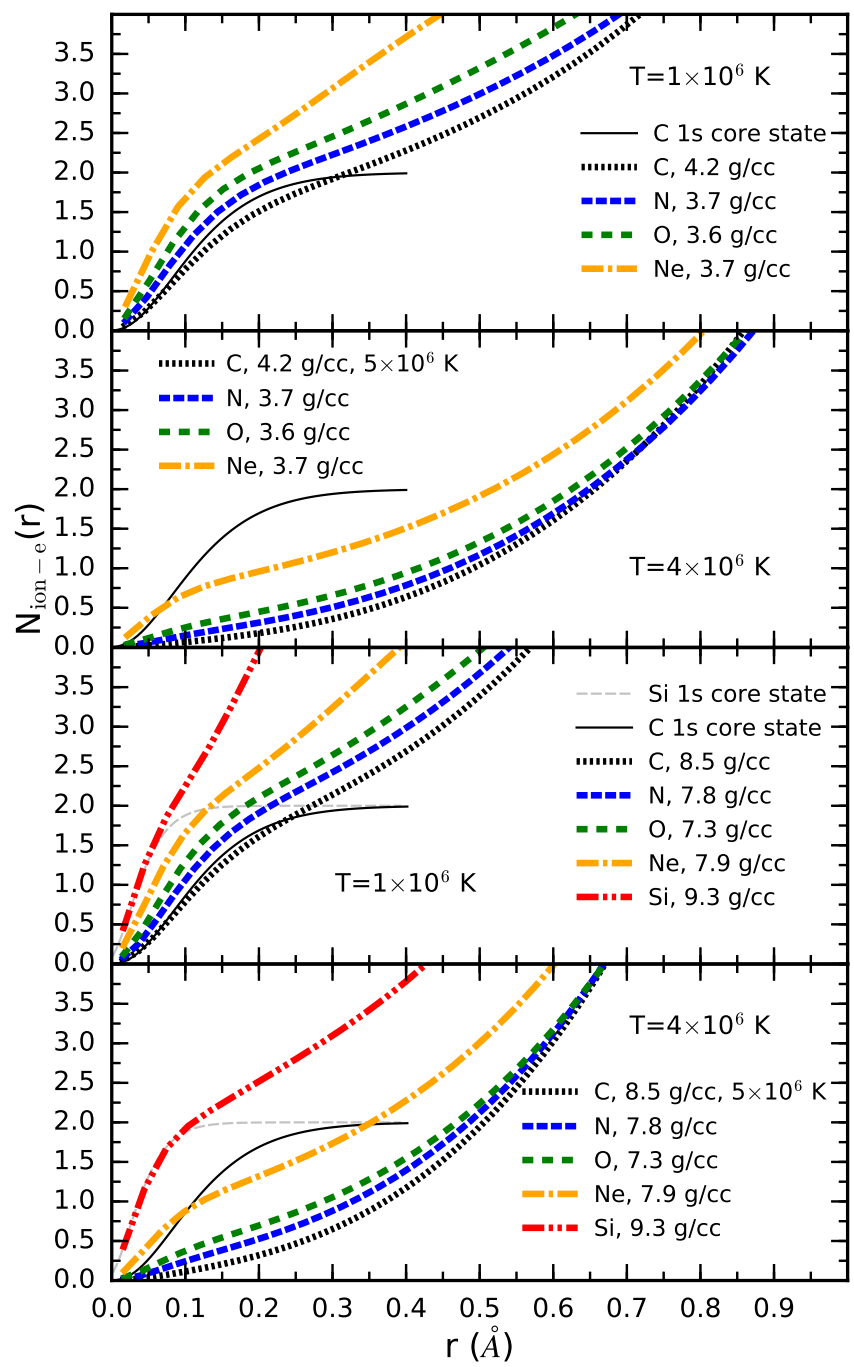

Figure 13: Number of electrons contained in a sphere of radius, r, around nuclei from various PIMC simulations of plasmas at two sets of temperatures and densities.

electron-electron correlations for pairs with opposite spins. The functions are multiplied by the number density $\rho$, so that the integral under the curves is proportional to the number of electrons. Similar to the earlier discussion of $\mathrm{C}$ and $\mathrm{H}_{2} \mathrm{O}$, the electrons are most highly correlated for low temperatures, which reflects that multiple electrons occupy bound states around a given nucleus. Higher $\mathrm{Z}$ elements are more highly correlated than low Z elements at low temperature due to their larger electron binding energy. As temperature increases, electrons are thermally excited, decreasing the correlation among each other. It is again clear that higher $\mathrm{Z}$ systems require significantly higher temperatures to ionize core electrons. The increase in correlation with density at short distances, consistent with a lower ionization fraction, is more significant for lower $\mathrm{Z}$ elements in the density-temperature range under consideration.

Figure 15 shows electron-electron pair correlations in 


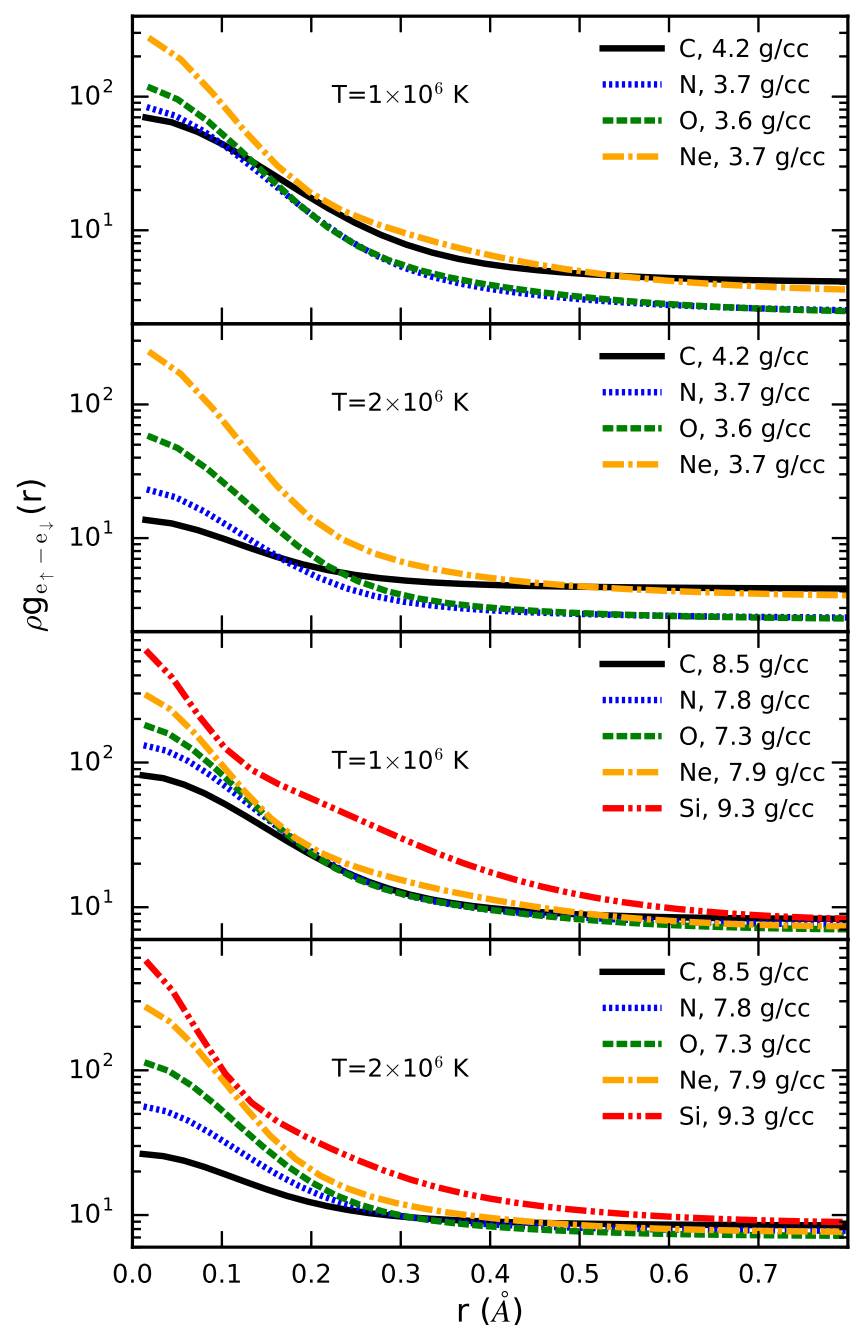

Figure 14: The electron-electron pair-correlation functions for electrons with anti-parallel spins in PIMC calculations of various plasmas at two sets of temperatures and densities.

C, N, O, Ne, and Si plasmas for electrons with parallel spins. The positive correlation at intermediate distances reflects that different electrons with parallel spins are bound to a given nucleus. Higher Z systems show a larger correlation at short distances since they binds core electrons more tightly. For short separations, electrons strongly repel due to Pauli exclusion and the functions decay to zero. As density increases, the correlation peak at intermediate distances decreases, which indicates the effect of pressure ionization on the $\mathrm{L}$ shell. The pressure ionization is more significant in larger $\mathrm{Z}$ systems at lower temperatures since more electrons are still bound and experiencing Pauli exclusion effects. As temperature increases, the correlation of lower $\mathrm{Z}$ systems more readily approaches that of an ideal fluid.

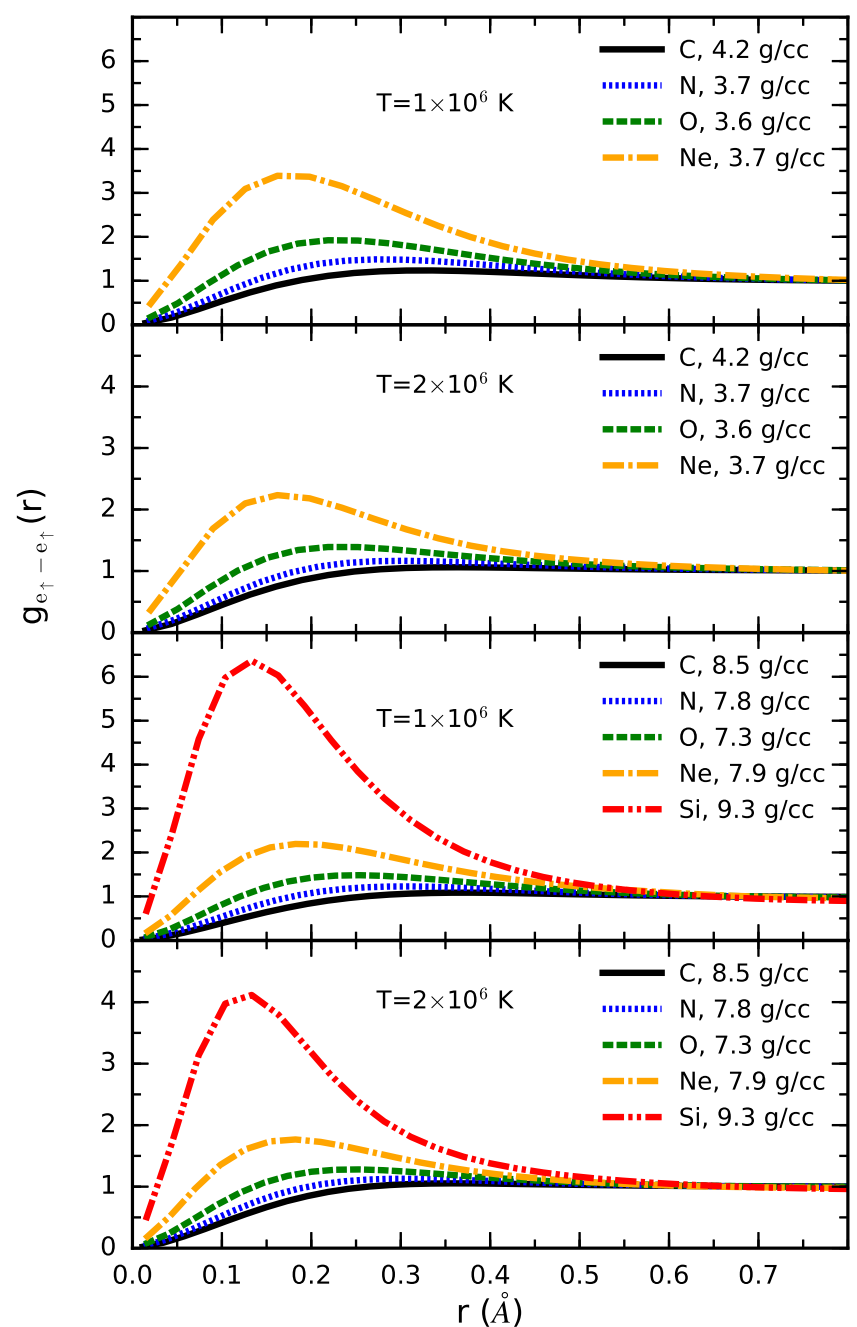

Figure 15: The electron-electron pair-correlation functions for electrons with parallel spins in PIMC calculations of various plasmas at two sets of temperatures and densities.

\section{Comparison of Shock Hugoniot Curves}

Dynamic shock compression experiments 36, 37, 38, 39, 40] allow one to measure the EOS and other physical properties of hot, dense fluids. Such experiments are often used to determine the principal Hugoniot curve, which is the locus of final states that can be obtained from different shock velocities. DFT-MD has been validated by experiments as an accurate tool for predicting the shock compression of a variety of different materials 41, 42], including nitrogen [43, 44].

In the course of a shock wave experiment, a material whose initial state is characterized by an internal energy, pressure, and volume $\left(E_{0}, P_{0}, V_{0}\right)$ will change to a final state denoted by $(E, P, V)$ while conserving mass, momentum, and energy. This leads to the Rankine-Hugoniot relation [45],

$$
\left(E-E_{0}\right)+\frac{1}{2}\left(P+P_{0}\right)\left(V-V_{0}\right)=0 .
$$




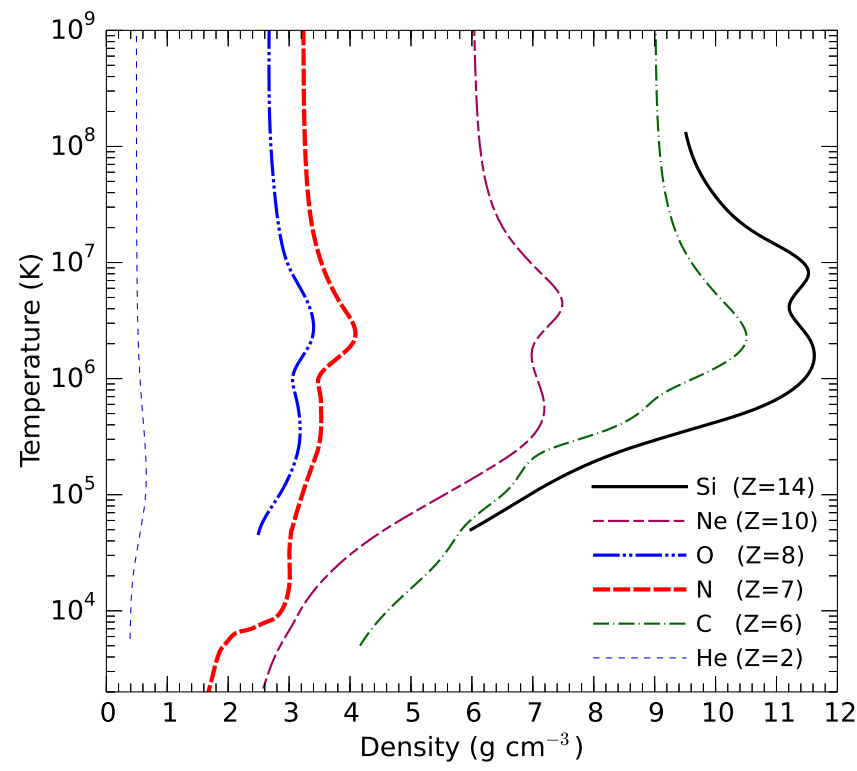

Figure 16: Hugoniot curves of various materials in temperaturedensity space at their initial ambient or experimental densities. The initial densities (in $\mathrm{g} \mathrm{cm}^{-3}$ ) are He: 0.124, C: 2.253, N 0.807, O: $0.667, \mathrm{Ne}: 1.507$, and Si: 2.329 .

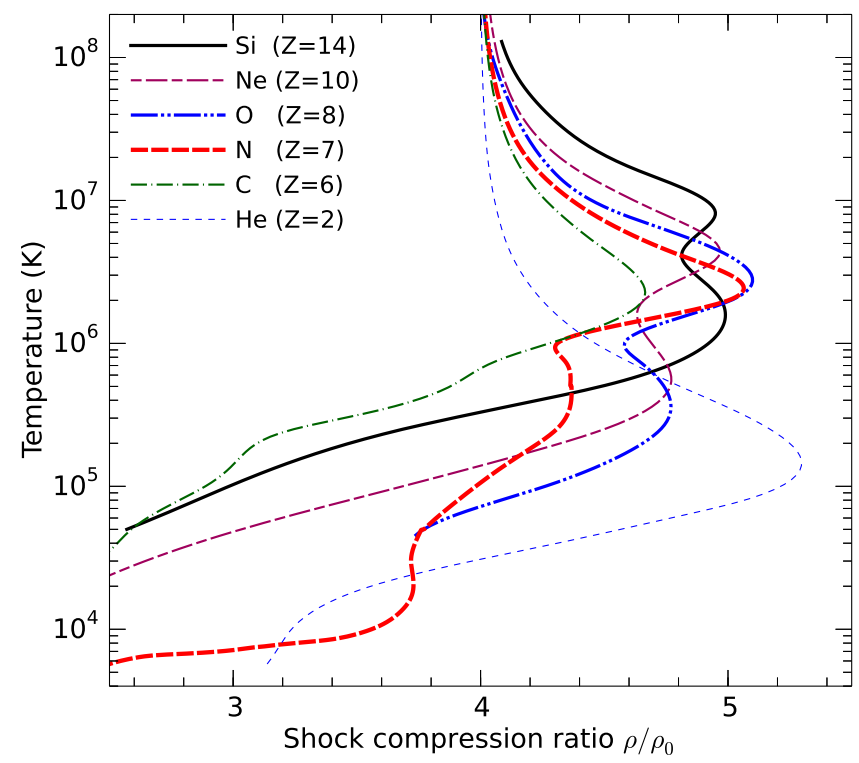

Figure 17: Temperature along the Hugoniot as a function of shock compression ratio.

Here, we compare the Hugoniots from the first-principles EOS data reported in our previous PIMC and DFT-MD simulations of $\mathrm{He}$ [15], C [16], N [17], O [18], Ne [19], and $\mathrm{Si}$ [21] in the WDM and plasma regimes. For each Hugoniot curve shown here, DFT-MD data is plotted for $\mathrm{T}<1 \times 10^{6} \mathrm{~K}$, and PIMC results are plotted for higher temperatures. Figure 16 compares the temperature dependence along the Hugoniot curve as a function of density for each system initialized at their ambient or experimental

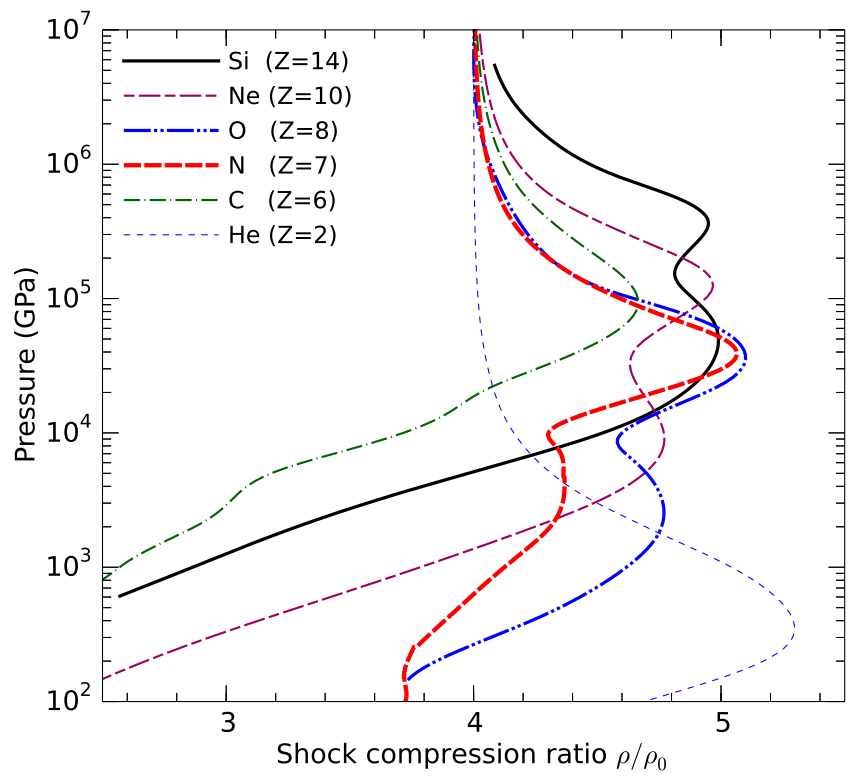

Figure 18: Pressure along the Hugoniot curve as a function of shock compression ratio.

densities (see the Fig. caption). The ordering of the curves depends strongly on the initial density and the atomic number, Z, of the system. Generally, systems with higher atomic numbers correspond to higher densities, but since the ambient densities may vary, the ordering of the curves does not always correspond to ordering of the atomic numbers of the systems.

Figures 17 and 18 compare the temperature and pressure dependence, respectively, along the Hugoniot curve as a functions of the shock-compression ratio for each system. In the high-temperature limit, all curves converge to a compression ratio of 4 , which is the value of a nonrelativistic, ideal gas. The magnitude of the shock compression ratio and the structure along the Hugoniot is determined by the excitation of internal degrees of freedom, such as dissociation and ionization processes, and the interaction effects, which directly depend on Z. Exciting internal degrees of freedom increases compression, while increased interaction effects decrease compression.

In both plots, each Hugoniot curve exhibits a distinct maxima in the shock compression corresponding to $\mathrm{K}$ or $\mathrm{L}$ shell ionization. The temperature at the compression maxima is directly determined by $\mathrm{Z}$ because the binding energy scales as $\mathrm{Z}^{2}$, which means a higher temperature is needed to reach the regime of ionization. Therefore, as $\mathrm{Z}$ increases, the compression peak temperatures increase.

However, the magnitude of the shock compression itself does not strictly increase with $\mathrm{Z}$ in our plots because systems with a higher initial density tend to have a smaller increase in the shock compression ratio due to stronger interaction effects. Therefore, the lower-initial-density systems (He, N, O, and $\mathrm{Ne}$ ) all have higher shock compression ratios than the higher-initial-density systems (C and $\mathrm{Si}$ ), 
particularly at lower pressure and temperature conditions. Indeed, the compression maxima are largest for the lowest initial densities. The differences in the shock-compression ratios are smaller among the systems near the compression maxima due to increased compression from more ionization effects in higher $\mathrm{Z}$ systems.

\section{Conclusion}

In conclusion, we have analyzed and compared results from PIMC and DFT-MD calculations for several firstand second-row materials in the liquid, warm dense matter, and plasma regimes. We have provided ionic and electronic pair-correlation functions for carbon and water plasmas and shown that they follow physical trends, such as increased ionization fraction with temperature and decreased K-shell ionization fraction with density. We then compared pair-correlation functions and Hugoniot curves for several materials (He, C, N, O, Ne, and $\mathrm{Si}$ ) comprised of increasing higher $\mathrm{Z}$ elements. The pair-correlation functions reveal how the plasma structure and ionization processes evolve with temperature and density. The paircorrelation functions and Hugoniot curves follow specific trends in temperature and density that depend directly on $\mathrm{Z}$ due to physical internal degrees of freedom and interaction effects, and, in the case of the Hugoniot curves, on initial density. Finally, we find the temperature of the compression maxima in the Hugoniot curves, associated with L-shell and K-shell ionization, increases directly with $\mathrm{Z}$, while the magnitude of the compression ratio depends mostly on the initial Hugoniot curve density.

\section{Acknowledgments}

This research is supported by the U. S. Department of Energy, grants DE-SC0010517 and DE-SC0016248. Computational support was provided by NERSC and the Janus supercomputer, which is supported by the National Science Foundation (Grant No. CNS-0821794), the University of Colorado, and the National Center for Atmospheric Research.

\section{References}

[1] R. Betti (Ed.), Advancing the Science of High Energy Density Laboratory Plasmas, Office of Fusion Energy Science (OFES)/Fusion Energy Science Advisory Committee (FESAC), Washington D.C., 2009.

[2] R. Rosner, D. Hammer, T. Rothman (Eds.), Basic Research Needs for high energy density laboratory physics, U.S. Department of Energy, Washington D.C., 2010.

[3] F. Graziani, M. P. Desjarlais, R. Redmer, S. B. Trickey (Eds.), Frontiers and Challenges in Warm Dense Matter, Springer International Publishing, 2014.

[4] M. Edwards, P. Patel, J. Lindl, L. Atherton, S. Glenzer, S. Haan, J. Kilkenny, O. Landen, E. Moses, A. Nikroo, et al., Progress towards ignition on the national ignition facility, Physics of Plasmas (1994-present) 20 (7) (2013) 070501.
[5] B. F. Rozsnyai, J. R. Albritton, D. A. Young, V. N. Sonnad, D. A. Liberman, Theory and experiment for ultrahigh pressure shock hugoniots, Physics Letters A 291 (4) (2001) 226-231.

[6] C. Pierleoni, D. M. Ceperley, B. Bernu, W. R. Magro, Equation of State of the Hydrogen Plasma by Path Integral Monte Carlo, Phys. Rev. Lett. 73 (1994) 2145.

[7] F. Lambert, J. Clérouin, G. Zérah, Very-high-temperature molecular dynamics, Phys. Rev. E 73 (2006) 016403.

[8] V. V. Karasiev, D. Chakraborty, O. A. Shukruto, S. Trickey, Nonempirical generalized gradient approximation free-energy functional for orbital-free simulations, Physical Review B 88 (16) (2013) 161108.

[9] T. Sjostrom, J. Daligault, Fast and accurate quantum molecular dynamics of dense plasmas across temperature regimes, Physical review letters 113 (15) (2014) 155006.

[10] P. Sterne, S. Hansen, B. Wilson, W. Isaacs, Equation of state, occupation probabilities and conductivities in the average atom purgatorio code, High Energy Density Physics 3 (1) (2007) 278282.

[11] B. F. Rozsnyai, Equation of state calculations based on the selfconsistent ion-sphere and ion-correlation average atom models, High Energy Density Physics 10 (2014) 16-26.

[12] C. Starrett, D. Saumon, Equation of state of dense plasmas with pseudoatom molecular dynamics, Physical Review E 93 (6) (2016) 063206.

[13] M. P. Surh, T. W. Barbee III, L. H. Yang, First principles molecular dynamics of dense plasmas, Physical review letters 86 (26) (2001) 5958.

[14] B. Militzer, D. M. Ceperley, Path intergral Monte Carlo simulation of low-density hydrogen plasma, Phys. Rev. E 63 (2001) 066404.

[15] B. Militzer, Path integral Monte Carlo and density functional molecular dynamics simulations of hot, dense helium, Phys. Rev. B 79 (2009) 155105.

[16] K. P. Driver, B. Militzer, All-electron Path Integral Monte Carlo Simulations of Warm Dense Matter: Application to Water and Carbon Plasmas, Phys. Rev. Lett. 108 (2012) 115502.

[17] K. P. Driver, B. Militzer, First-principles equation of state calculations of warm dense nitrogen, Phys. Rev. B 91 (2016) 064101.

[18] K. Driver, F. Soubiran, S. Zhang, B. Militzer, First-principles equation of state and electronic properties of warm dense oxygen, The Journal of chemical physics 143 (16) (2015) 164507.

[19] K. P. Driver, B. Militzer, First-principles simulations and shock Hugoniot calculations of warm dense neon, Phys. Rev. B 91 (2015) 045103.

[20] See S. Zhang, K. P. Driver, F. Soubrian, and B. Milizer, "Path Integral Monte Carlo Studies of Warm Dense Sodium" within this proceeding.

[21] B. Militzer, K. P. Driver, Development of path integral monte carlo simulations with localized nodal surfaces for second-row elements, Phys. Rev. Lett 115 (2015) 176403.

[22] D. Ceperley, Fermion nodes, Journal of statistical physics 63 (56) (1991) 1237-1267.

[23] D. M. Ceperley, Path integrals in the theory of condensed helium, Rev. Mod. Phys. 67 (1995) 279.

[24] D. M. Ceperley, Path integral Monte Carlo Methods for Fermions, in: K. Binder, G. Ciccotti (Eds.), Monte Carlo and Molecular Dynamics of Condensed Matter Systems, Vol. 49, Editrice Compositori, Bologna, Italy, 1996, p. 443.

[25] D. Marx, J. Hutter, Ab initio molecular dynamics: Theory and implementation, in: J. Grotendorst (Ed.), Modern Methods and Algorithms of Quantum Chemistry Proceedings, Vol. 3, NIC Series, Julich, Germany, 2000, pp. 301-449.

[26] M. Koenig et al., Progress in the study of warm dense matter, Plasma Phys. Contr. F. 47 (2005) B441.

[27] E. L. Pollock, Properties and Computation of the Coulomb Pair Density Matrix, Comput. Phys. Commun. 52 (1988) 49.

[28] B. Militzer, R. L. Graham, Simulations of dense atomic hydrogen in the wigner crystal phase, J. Phys. Chem. Solids 67 (2006) 2136. 
[29] B. Militzer, Computation of the high temperature coulomb density matrix in periodic boundary conditions, Computer Physics Communications 204 (2016) 88-96.

[30] D. M. Ceperley, Fermion Nodes, J. Stat. Phys. 63 (1991) 1237.

[31] E. W. Brown, B. K. Clark, J. L. DuBois, D. M. Ceperley, PathIntegral Monte Carlo Simulations of the Warm Dense Homogeneous Electron Gas, Phys. Rev. Lett. 110 (2013) 146405.

[32] D. N. Mermin, Thermal properties of the inhomogeneous electron gas, Phys. Rev. 137 (1965) A1441.

[33] G. Kresse, J. Furthmüller, Efficient iterative schemes for $a b$ initio total-energy calculations using a plane-wave basis set, Phys. Rev. B 54 (1996) 11169.

[34] P. E. Blöchl, Projector augmented-wave method, Phys. Rev. B 50 (1994) 17953.

[35] J. P. Perdew, K. Burke, M. Ernzerhof, Generalized gradient approximation made simple, Phys. Rev. Lett. 77 (1996) 3865.

[36] M. Bastea, A. C. Mitchell, W. J. Nellis, High Pressure InsulatorMetal Transition in Molecular Fulid Oxygen, Phys. Rev. Lett. 86 (2001) 3108.

[37] R. Chau, a. Mitchell, R. Minich, W. Nellis, Metallization of Fluid Nitrogen and the Mott Transition in Highly Compressed Low-Z Fluids, Phys. Rev. Lett. 90 (2003) 245501.

[38] R. Jeanloz, P. M. Celliers, G. W. Collins, J. H. Eggert, K. K. Lee, R. S. McWilliams, S. Brygoo, P. Loubeyre, Achieving highdensity states through shock-wave loading of precompressed samples, Proceedings of the National Academy of Sciences 104 (22) (2007) 9172-9177.

[39] R. Smith, J. Eggert, R. Jeanloz, T. Duffy, D. Braun, J. Patterson, R. Rudd, J. Biener, A. Lazicki, A. Hamza, et al., Ramp compression of diamond to five terapascals, Nature 511 (7509) (2014) 330-333.

[40] H. Kishimura, H. Matsumoto, N. Thadhani, Effect of shock compression on single crystalline silicon, in: Journal of Physics: Conference Series, Vol. 215, IOP Publishing, 2010, p. 012145.

[41] S. Root, R. J. Magyar, J. H. Carpenter, D. L. Hanson, T. R. Mattsson, Shock Compression of a Fifth Periodic Element: Liquid Xenon to 840 GPa, Phys. Rev. Lett. 105 (2010) 085501.

[42] T. R. Mattsson, S. Root, A. E. Mattsson, L. Shulenburger, R. J. Magyar, D. G. Flicker, Validating density-functional theory simulations at high energy-density conditions with liquid krypton shock experiments to $850 \mathrm{GPa}$ on Sandia's Z machine, Phys. Rev. B 90 (2014) 184105.

[43] J. D. Kress, S. Mazevet, L. A. Collins, W. W. Wood, Densityfunctional calculation of the Hugoniot of shocked liquid nitrogen, Phys. Rev. B 61 (2000) 024203.

[44] S. Mazevet, J. Johnson, J. Kress, L. Collins, P. Blottiau, Density-functional calculation of multiple-shock Hugoniots of liquid nitrogen, Phys. Rev. B 65 (2001) 014204.

[45] Y. B. Zeldovich, Y. P. Raizer, Elements of Gasdynamics and the Classical Theory of Shock Waves, Academic Press, New York, 1968 